\title{
Magnetic and structural phase diagram of the solid solution $\mathrm{LaCo}_{x} \mathrm{Mn}_{1-x} \mathrm{O}_{3}$
}

\author{
C. L. Bull, H. Y. Playford, K. S. Knight, G. B. G. \\ Stenning, and M. G. Tucker
}

\section{Published version information}

Citation: Bull, $\mathrm{CL}$ et al. "Magnetic and structural phase diagram of the solid solution LaCoxMn1-xO3." Physical Review B, vol. 94, no. 1 (2016): 014102.

doi: $10.1103 /$ PhysRevB.94.014102

This version is made available in accordance with publisher policies. Please cite only the published version using the reference above. 


\title{
Magnetic and Structural Phase Diagram of the Solid Solution $\operatorname{LaCo}_{x} \mathrm{Mn}_{1-x} \mathrm{O}_{3}$
}

\author{
C. L. Bull, ${ }^{1, *}$ H. Y. Playford,${ }^{1}$ K. S. Knight,${ }^{1,2,3}$ G. B. G. Stenning, ${ }^{1}$ and M. G. Tucker ${ }^{1,4}, \dagger$ \\ ${ }^{1}$ STFC ISIS Facility, Rutherford Appleton Laboratory, Didcot, OX11 0QX, UK \\ ${ }^{2}$ Department of Earth Sciences, The Natural History Museum, Cromwell Road, London, SW $5 B D$, UK \\ ${ }^{3}$ Department of Earth Sciences, University College London, Gower Street, London, WC1E 6BT, UK \\ ${ }^{4}$ Diamond Light Source Ltd, Harwell Oxford, Didcot, OX11 ODE, UK
}

(Dated: June 13, 2016)

\begin{abstract}
We present a structural and magnetic phase diagram of the solid solution $\mathrm{LaCo}_{x} \mathrm{Mn}_{1-x} \mathrm{O}_{3}$. We show by neutron diffraction that the monoclinic structure previously observed for the elpasolite form $\mathrm{La}_{2} \mathrm{CoMnO}_{6}\left(\mathrm{LaCo}_{0.5} \mathrm{Mn}_{0.5} \mathrm{O}_{3}\right)$ is also observed for another member of the solid solution $x=$ 0.35. We also present the transition temperatures for the orthorhombic/monoclinic structures of the series to the rhombohedral structure and determine the expected transition temperatures from rhombohedral to cubic symmetry. We present the magnetic structures as determined by neutron diffraction for materials with lower cobalt content and provide evidence, including AC and DC susceptibility measurements, for the possible glassy nature of the magnetism in the cobalt-rich materials in the series. Based on high-resolution neutron diffraction, we also suggest that there is a limit to the $\mathrm{LaCo}_{x} \mathrm{Mn}_{1-x} \mathrm{O}_{3}$ solid solution at $x=0.85$. Finally we present a possible, previously unreported, low temperature monoclinic structure for the sample $\mathrm{LaCo}_{0.75} \mathrm{Mn}_{0.25} \mathrm{O}_{3}$.
\end{abstract}

PACS numbers: 61,75

\section{INTRODUCTION}

Perovskites with the general formula $\mathrm{ABO}_{3}$ (where $\mathrm{A}$ is often a lanthanide and $\mathrm{B}$ commonly a transition metal) are an important and widely-studied class of materials, characterised by subtle structural distortions from the cubic aristotype (characterised by space group $P m \overline{3} m$ ). The ideal perovskite structure consists of a corner-shared network of $\mathrm{BO}_{6}$ octahedra with the larger A cations sitting in interstitial sites co-ordinated by $12 \mathrm{O}$ atoms. The enormous variety of physical and chemical properties that the perovskite family displays arises from the fact that the $\mathrm{A}$ and $\mathrm{B}$ cations can be selected from a large proportion of the periodic table.

Double perovskites (elpasolite) represent a variant of the perovskite structure with the general formula $\mathrm{A}_{2} \mathrm{BB}^{\prime} \mathrm{X}_{6}$ in which the prime indicates the possibility of different cations. In general the B-site cations determine the physical properties of the double perovskites and three B-cation sublattice types are recognised; random, rock-salt, and layered, with the latter two demonstrating an ordered arrangement of the resulting $\mathrm{BX}_{6}$ octahedra. B-site ordering in double perovskites typically occurs when there is a significant charge and/or size difference between the B and B' cations.

The solid solution $\mathrm{LaCo}_{x} \mathrm{Mn}_{1-x} \mathrm{O}_{3}$ has been the subject of a large body of work focused on understanding its complex structure-property relationships. It is closely related to the A-site doped manganites which famously exhibit colossal magnetoresistance, ${ }^{1}$ and itself displays a wealth of interesting magnetic and electronic properties

\footnotetext{
* craig.bull@stfc.ac.uk

$\dagger$ Now at: Spallation Neutron Source, One Bethel Valley Road, MS-6475, Oak Ridge, TN, USA
}

which change as a function of $x$. Furthermore, mixedmetal perovskite oxides are increasingly of interest for catalytic applications. $^{2-4}$ For example, it has been shown that low-level doping of $\mathrm{LaCoO}_{3}$ with $\mathrm{Mn}$ forms an effective catalyst for the oxidation of particulate matter in vehicle exhaust. ${ }^{5}$

The $x=1$ end member, $\mathrm{LaCoO}_{3}$, undergoes some well characterised temperature driven electronic transitions. At low temperature $(<50 \mathrm{~K}), \mathrm{LaCoO}_{3}$ is a non-magnetic insulator with the trivalent cobalt in the low spin (LS) state configuration $t_{2 g}{ }^{6} e_{g}{ }^{0}$. At $\sim 100 \mathrm{~K}$ the material becomes paramagnetic and with increasing temperature the high-spin (HS) $t_{2 g}{ }^{4} e_{g}{ }^{2}$ configuration is populated. Above $648 \mathrm{~K}$ charge transfer between spin states occurs and disproportionation into $\mathrm{Co}^{2+}$ and $\mathrm{Co}^{4+}$ species is observed. ${ }^{6}$ Structurally $\mathrm{LaCoO}_{3}$ is rhombohedral, space group $R \overline{3} c\left(a^{-} a^{-} a^{-}\right.$in Glazer notation, representing three antiphase tilts around the cubic $a, b$ and $c$ axes). ${ }^{7,8}$ In contrast at the other end of the series $\mathrm{LaMnO}_{3}(x=0)$ is an antiferromagnet (Néel temperature $140 \mathrm{~K}$ ), an insulator, and crystallizes in an orthorhombic structure (space group Pbnm). ${ }^{9}$ This hettotype structure results from anti-phase rotations of the $\mathrm{MnO}_{6}$ octahedra about the $[100]$ cubic/[010]cubic directions and in-phase rotations around the [001]cubic direction, and can be described by the $a^{-} a^{-} c^{+}$tilt system in Glazer notation. ${ }^{8}$

For members of the solid solution rich in cobalt $(x>$ 0.6 ), the rhombohedral structure dominates, whereas for $x<0.6$ the orthorhombic structure is found (for $x=$ 0.6 a biphasic mixture is observed). ${ }^{10}$ The structure of the material with $x=0.5$ has been a matter of some debate over the years and has been reported as cubic, ${ }^{11}$ orthorhombic, ${ }^{12}$ or even biphasic in nature. ${ }^{13}$ However, high resolution neutron diffraction data show that the sample crystallizes in the monoclinic space group $P 2_{1} / n$ 
(tilt system $a^{-} a^{-} c^{+}$) which is a maximal non-isomorphic subgroup of the orthorhombic Pbnm. In the monoclinic structure, the manganese and cobalt cations order at the $90 \%$ level on the $2 b$ and $2 c$ sites, respectively. ${ }^{14}$

For the $\mathrm{LaCo}_{x} \mathrm{Mn}_{1-x} \mathrm{O}_{3}$ solid solution there have been numerous magnetic property studies and in 1961 Goodenough et al. ${ }^{13}$ suggested that across the series the cobalt ions were in the diamagnetic low-spin state $\mathrm{Co}^{3+}$ and hence only the ferromagnetic $\mathrm{Mn}^{3+}-\mathrm{O}^{2-}$ $\mathrm{Mn}^{3+}$ and $\mathrm{Mn}^{3+}-\mathrm{O}^{2-}-\mathrm{Mn}^{4+}$ interactions contributed to the magnetic properties. However, further magnetic and electrical characterisation studies by Jonker showed that there is also a contribution from $\mathrm{Mn}^{4+}$ $\mathrm{O}^{2-}-\mathrm{Co}^{2+}, \mathrm{Mn}^{3+}-\mathrm{O}^{2-}-\mathrm{Mn}^{3+}$ and $\mathrm{Mn}^{3+}-\mathrm{O}^{2-}-\mathrm{Mn}^{4+}$ superexchange interactions through the anions. ${ }^{15}$ Work by Troyanchuk et al. ${ }^{16}$ found that the magnetic properties of $\mathrm{LaCo}_{x} \mathrm{Mn}_{1-x} \mathrm{O}_{3}$ could be explained on the basis of positive $\mathrm{Mn}^{3+}-\mathrm{Mn}^{4+}, \mathrm{Mn}^{3+}-\mathrm{Mn}^{3+}, \mathrm{Mn}^{4+} \mathrm{Co}^{2+}$ and negative $\mathrm{Mn}^{4+}-\mathrm{Mn}^{4+}, \mathrm{Co}^{2+}-\mathrm{Co}^{2+}, \mathrm{Co}^{2+}-\mathrm{Mn}^{3+}$ superexchange interactions combined with $\mathrm{Mn}^{4+}$ and $\mathrm{Co}^{2+}$ cation ordering. In a more recent study combining both magnetic and transport properties it was shown that for the orthorhombic manganese-rich region cobalt enters as $\mathrm{Co}^{2+}$ and induces a mixed $\mathrm{Mn}^{3+} / \mathrm{Mn}^{4+}$ valence state in the manganese. For $x \approx 0.4$ the majority of the ions are $\mathrm{Co}^{3+}$ and $\mathrm{Mn}^{3+}$, and in the rhombohedral cobaltrich region the manganese is present preferentially in the $\mathrm{Mn}^{4+}$ state, inducing electron-like carriers in the Co-O subarray. ${ }^{10}$ In contrast with this, Sikora et al. ${ }^{17}$ found from X-ray absorption spectroscopy that the valence of both cations increases almost linearly with $x$, and furthermore that they are mixed valent across the entire solid solution.

The bulk magnetisation behaviour of these materials reflects the complexity of the underlying interactions. For $0.4 \leq x \leq 0.9$, Troyanchuk et al. ${ }^{16}$ proposed a mixture of cationically ordered ferromagmetic and disordered spin-glass regions, and noted that samples quenched from high temperature had an increased fraction of spin-glasslike domains. Autret et al..$^{10}$ observed a crossover from predominantly ferromagnetic interactions at low Co content to predominantly antiferromagnetic interactions at $x=1$. For $x=0.4$, a two-step transition to a bulk ferromagnetic phase has been observed and attributed to the presence of ferromagnetic clusters forming at the higher transition temperature and coalescing to produce long range order (observable by neutron diffraction) at the lower. Whether these clusters result from intrinsic chemical inhomogeneity or from the presence of distinct cation ordered and disordered regions is the subject of some debate. ${ }^{10,18}$ For $x=0.5$, two ferromagnetic phases have been reported: one with a higher $\mathrm{T}_{\mathrm{C}}(\sim 225 \mathrm{~K})$ which contains exclusively $\mathrm{Co}^{2+}$ and $\mathrm{Mn}^{4+}$ and exhibits extensive cation ordering; and a second with a lower $\mathrm{T}_{\mathrm{C}}$ $(\sim 150 \mathrm{~K})$ which contains a disordered arrangement of mixed valent cations. ${ }^{19,20}$ The coexistence of these phases is almost unavoidable, though the fraction of each can be controlled by the preparation method followed. ${ }^{19,21}$ It is therefore perhaps unsurprising that a range of magnetic ground-states has been proposed, from long range ferromagnetic order, ${ }^{22}$ to cluster-glass models, ${ }^{23}$ and even superparamagnetism. ${ }^{24}$ For $x=0.8$, a similar, though less pronounced, non-uniform magnetic behaviour was observed and was attributed to the existence of small ferromagnetic clusters in a disordered matrix. ${ }^{10}$ Furthermore, a peak in the magnetic susceptibility at $25 \mathrm{~K}$ was attributed to a spin-glass transition.

We have synthesised and studied materials in the $\mathrm{LaCo}_{x} \mathrm{Mn}_{1-x} \mathrm{O}_{3}$ series (with $x=0.2,0.35,0.5,0.75$ and 0.9 ). Based upon these studies we present a new structural phase diagram for this solid solution. We show that crystallographic ordering of the $\mathrm{Co} / \mathrm{Mn}$ ions occurs at room temperature not only for $x=0.5$ but also for $x=0.35$, and we suggest that the sample with $x=0.75$ undergoes a rhombohedral to monoclinic phase transition at $\sim 100 \mathrm{~K}$. We have also determined temperatures for the high-temperature rhombohedral to cubic transition for each sample studied. We have used neutron diffraction and magnetometry to analyse the magnetic structure of all the materials in the series and present evidence for a glassy ferromagnetic behaviour in the cobalt-rich samples.

\section{EXPERIMENTAL}

\section{A. Synthesis and X-ray Characterisation}

Polycrystalline samples of $\mathrm{LaCo}_{x} \mathrm{Mn}_{1-x} \mathrm{O}_{3}$ were prepared by a modified nitrate decomposition route. Appropriate mixtures of hydrated metal nitrate salts were dissolved in the minimum amount of hot water in an alumina crucible, and decomposition was achieved first by heating to dryness on a hotplate and then by a slow heating to $870 \mathrm{~K}$ in a tube furnace in air. Samples were subsequently annealed at $1270 \mathrm{~K}$ for 12 hours, and were cooled at a rate of $\sim 2 \mathrm{~K} \mathrm{~min}^{-1}$ again in an air atmosphere. The degree of phase purity of the perovskite was determined by X-ray diffraction (XRD) with a Rigaku Miniflex X-ray diffractometer using $\mathrm{Cu} \mathrm{K}_{\alpha 1,2}$ radiation. High-temperature X-ray diffraction was carried out on a Rigaku SmartLab diffractometer fitted with a germanium (220) monochromator. An Anton Paar HTK hightemperature stage was used to collect diffraction patterns in the temperature range $293-1200 \mathrm{~K}$.

\section{B. Variable Temperature Neutron Diffraction}

Ambient and low-temperature time-of-flight neutron diffraction measurements were carried out on the Polaris and HRPD instruments at ISIS, the UK's spallation neutron source at the Rutherford Appleton Laboratory. Initially, room-temperature and $50 \mathrm{~K}$ diffraction patterns were collected from all samples using the mediumresolution, high-flux Polaris diffractometer (equipped 
with an Abingdon Scientific (AS) "Orange" cryostat for the low temperature measurements). Subsequent detailed characterisation of the cobalt-rich end of the series was carried out on the high-resolution HRPD instrument at temperatures in the range $4.2-300 \mathrm{~K}$ again using an AS "Orange" cryostat.

\section{Magnetic Characterisation}

DC magnetisation measurements as a function of temperature and applied magnetic field were carried out using a Quantum Designs MPMS-XL7 SQUID magnetometer. A finely ground powder sample of known mass was placed into a gelatine capsule and mounted into a straw before being introduced into the instrument at $300 \mathrm{~K}$. Measurements of the DC magnetisation were made as a function of temperature $(1.8-300 \mathrm{~K})$ in an applied field of 100 Oe and in both field cooled (FC) and zerofield cooled (ZFC) conditions. Hysteresis loops were also measured $( \pm 5 \mathrm{~T})$ as a function of temperature in the range $10-250 \mathrm{~K}$.

AC magnetic susceptibility was measured using a Quantum Designs PPMS-9 equipped with an AC measurement system (ACMS). The powdered sample was pressed into a pellet and annealed at $1100{ }^{\circ} \mathrm{C}$ for $\sim 8$ hours prior to loading into a straw as described above. Magnetization measurements at various temperatures (2 - $300 \mathrm{~K}$ ) were carried out in zero field cooled upon warming (ZFCW) conditions, with DC bias fields between 0 and $1000 \mathrm{Oe}$, an $\mathrm{AC}$ field magnitude of $10 \mathrm{Oe}$ and at a range of frequencies between $250-10000 \mathrm{~Hz}$.

\section{RESULTS AND DISCUSSION}

\section{A. X-ray Characterisation}

The samples were shown by Rietveld refinement to be on the whole single phase excepting a small contaminant of $\mathrm{La}_{2} \mathrm{O}_{3}(\sim 2-3 \mathrm{wt} \%)$. X-ray diffraction confirmed the crystallographic symmetry (within instrument and technique resolution) to be orthorhombic $(\mathrm{Pbnm})$ for $x=0.2$ and 0.35 samples, monoclinic $\left(P 2_{1} / n\right)$ for the $x=0.5$ sample and rhombohedral $(R \overline{3} c)$ for the 0.75 and 0.9 samples. Despite extensive efforts we were unable to prepare a phase pure sample with the composition of $x=0.6$ and can confirm previous reports that $\mathrm{a} \simeq 50: 50$ mixture of rhombohedral and orthorhombic phases are present. ${ }^{10}$ The relative proportion of Co and $\mathrm{Mn}$ in each sample was confirmed to lie within error of the intended stoichiometry using X-ray Fluorescence (XRF).

\section{B. X-ray Characterisation at High Temperature}

The high-temperature structural behaviour of all samples in the series has been studied using XRD. Changes
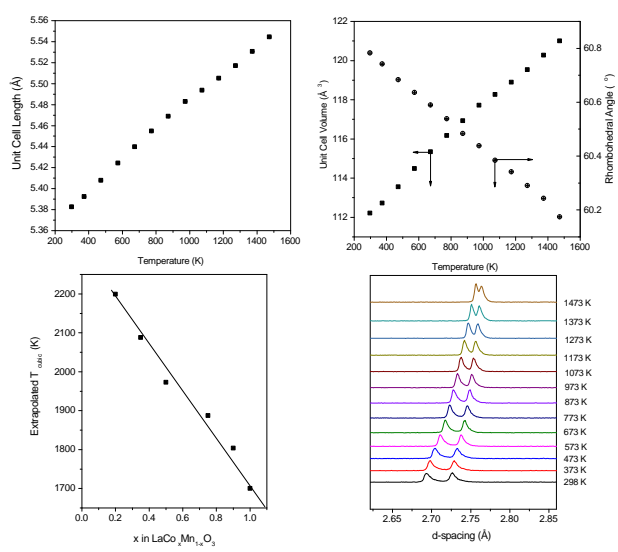

FIG. 1. Upper: The variation of determined unit cell length, volume and rhombohedral cell angle with temperature for the $x=0.9$ sample. Lower left: The extrapolated transition from the rhombohedral symmetry to cubic symmetry as a function of $x$. The changes upon heating in the x-ray diffraction pattern of the rhombohedral sample $\mathrm{LaCo}_{0.9} \mathrm{Mn}_{0.1} \mathrm{O}_{3}$ are shown in the lower right panel. The 211 and 101 reflections are shown at $\sim 2.69$ and $2.72 \AA$ respectively and are shown to shift to higher d-spacing with increasing temperature and also the spacing between the reflections is decreasing with increasing temperature.

are observed in the region of the 112, 020 and 200 reflections at around $\sim 2.75 \AA$ in the orthorhombic/monoclinic phases $(x=0.2,0.35$ and 0.5$)$ with increasing temperature as previously reported for $x=0.33 .{ }^{25}$ Indexing of the high temperature diffraction pattern above $573 \mathrm{~K}$ shows that the reflections are consistent with rhombohedral symmetry (indexed with the $R \overline{3} c$ space group in the rhombohedral metric in the case of $x=0.2$ and 0.35 and $R \overline{3}$ for $x=0.5$ ). Shown in the lower right panel of Figure 1 is the evolution of the 211 and 101 reflections for the rhombohedral $\mathrm{LaCo}_{0.9} \mathrm{Mn}_{0.1} \mathrm{O}_{3}$, which move to higher d-spacing as a result of thermal expansion of the unit cell and whose separation decreases with increasing temperature, as a result of the decrease of the rhombohedral unit cell angle. Diffraction patterns measured at $473 \mathrm{~K}$ show that there is co-existence of the orthorhombic and rhombohedral phases, as would be expected given the first order nature of the structural transition. ${ }^{26}$ The refined lattice parameters, unit cell volumes and rhombohedral unit cell angles are shown in the upper panels of Figure 1. Similar behaviour has been observed for all of the samples in the series which at ambient conditions are orthorhombic or monoclinic in that they transform to rhombohedral upon warming. The samples in the series which are rhombohedral at room temperature show no phase transitions in the temperature region measured using XRD, however they do show the same trend in that the splitting of the 211 and 101 reflections decreases with increasing temperature (the determined unit cell parameters for $x=0.9$ are shown in Figure 1). Assuming a 
continued linear behaviour of the rhombohedral alpha angle it is possible to extrapolate the temperature at which it would become $60^{\circ}$. With a alpha angle of $60^{\circ}$ in the rhombohedral symmetry it is assumed that the crystallographic symmetry would be transformed to the cubic $P m \overline{3} m$ via a second order phase transition as has been shown previously for other systems such as $\mathrm{LaAlO}_{3}$ and $\mathrm{PrAlO}_{3} \cdot{ }^{27,28}$ Figure 1 also shows the variation with $x$ of the temperature at which the rhombohedral angle extrapolates to 60 degrees. It is clear that with increasing cobalt content the temperature at which the system becomes metrically cubic decreases. Recently, Orayech et al proposed to have observed pseudo cubic symmetry $(F m \overline{3} m)$ in $\mathrm{La}_{2} \mathrm{CoMnO}_{6}$ at $1545 \mathrm{~K}$ (however that study may have been resolution limited). ${ }^{22}$ Also plotted is the extrapolated temperature at which $\mathrm{LaCoO}_{3}$ is expected to become cubic based upon the work of Thornton et $a l .{ }^{7}$. However, this is in contrast to the work recently published by Kobayashi et al. ${ }^{29}$ who suggest a pseudocubic transition at $1610 \mathrm{~K}$ (however it may be that their result is again resolution limited).

\section{Magnetic Characterisation}

Bulk DC magnetisation measurements have been performed for each of the samples in the series and the results are shown in Figures 2 and 3. For $x=0.2,0.35$ and 0.5 (Figure 2), the ZFCW and FCW magnetisation curves are consistent with the onset of long-range magnetic ordering. We see no evidence for the two-step transition previously reported for $x=0.4$ and $0.5 .^{10,18,21,24}$ Above $\mathrm{T}_{\mathrm{C}}$ the materials obey the Curie-Weiss law and the fitting to the linear region of an inverse susceptibility versus temperature plot allows the determination of an effective paramagnetic moment, $\mu_{\text {eff }}$ (Table I). The values of $\mu_{\text {eff }}$ are larger than the theoretical spin-only moments (even if both cations are assumed to be in a high spin configuration) and can be explained if the $3 \mathrm{~d}$ orbital contribution to the magnetic moment of $\mathrm{Co}^{2+}$ is not fully quenched. This is consistent with our previous report on the $x=0.33$ composition $\left(\mu_{\mathrm{eff}}=5.33 \mu_{\mathrm{B}} /\right.$ f.u. $),{ }^{25}$ and with the findings of Burnus et al. ${ }^{20}$ for $x=0.5$. The Weiss constants, $\theta_{W}$, obtained from the $x$-intercepts of the linear fits, are also shown in Table I. The positive values indicate that the magnetic interactions in the materials are predominantly ferromagnetic, and their similarity to $\mathrm{T}_{\mathrm{C}}$ for $x \leq 0.5$ suggest that these systems are not frustrated. Hysteresis loops ( $\mathrm{M}$ versus $\mathrm{H}$ ) are also shown in Figure 2, and are again consistent with the presence of ferromagnetic order at temperatures below $T_{C}$. The hysteresis loop at $100 \mathrm{~K}$ reveals an apparent saturation magnetisation of $2 \mu_{\mathrm{B}}$ per formula unit, which agrees reasonably well with the value obtained from $\mathrm{Ri}$ etveld refinement of neutron diffraction data collected at $50 \mathrm{~K}$ (see Section IIID), but is much smaller than the paramagnetic $\mu_{\text {eff }}$. This observation suggests that the ferromagnetic ordering is incomplete, perhaps because of the competing interactions and the inherent disorder of the system.

Significantly different magnetic behaviour is observed for the samples with $x=0.75$ and 0.9 , as shown in Figure 3. In general the response of these samples is much weaker than for the lower Co content materials, and furthermore the ZFCW curves show two distinct features, one at $\sim \mathrm{T}_{\mathrm{C}}$, and a second at $\sim 25-30 \mathrm{~K}$. The hysteresis loops measured below $145 \mathrm{~K}$ are reminiscent of those for a ferromagnetic material but do not approach saturation even at high field. At $145 \mathrm{~K}$ no hysteresis is seen and little change is observed on further warming. The values of $\mu_{\text {eff }}$ for these materials is significantly lower than for the $x \leq 0.5$ samples, and is consistent with a combination of $\mathrm{Mn}^{4+}$ and mixed valent $\mathrm{Co}$ in an intermediate spin (IS) state (calculated spin-only moments are 3.34 and $3.01 \mu_{B}$ for $x=0.75$ and 0.9 respectively). There is, of course, much controversy surrounding the assignment of an IS state in $\mathrm{LaCoO}_{3},{ }^{7,30,31}$ and given the complexity of the cation distribution and magnetic interactions in $\mathrm{LaCo}_{x} \mathrm{Mn}_{1-x} \mathrm{O}_{3}$ it is not possible to unambiguously assign oxidation or spin states. The observed moments could also be explained by a mixture of HS and LS states for either or both cations. The values of $\theta_{W}$ for these samples are rather smaller than $\mathrm{T}_{\mathrm{C}}$, which may suggest some degree of frustration is present in these materials. Further discussion of the unusual magnetic behaviour of the $x=0.75$ and 0.9 samples is given in Section IIID 2 .

TABLE I. Magnetic parameters for $\mathrm{LaCo}_{x} \mathrm{Mn}_{1-x} \mathrm{O}_{3}$

\begin{tabular}{lllll}
\hline \hline Sample & $\mathrm{T}_{\mathrm{C}}(\mathrm{K})^{\mathrm{a}}$ & $\mu_{\text {eff }}\left(\mu_{\mathrm{B}}\right)$ & $\theta_{W}(\mathrm{~K})$ & Ordered moment $\left(\mu_{\mathrm{B}}\right)^{\mathrm{b}}$ \\
$x=0.2$ & 177 & 4.99 & 186.36 & $2.81(1)$ \\
$x=0.35$ & 208 & 5.77 & 216.34 & $3.01(2)$ \\
$x=0.5$ & 228 & 5.33 & 230.38 & $3.14(1)$ \\
$x=0.75$ & 222 & 3.74 & 185.67 & - \\
$x=0.9$ & 224 & 2.91 & 143.57 & - \\
\hline \hline
\end{tabular}

a Defined as a minimum in the first derivative of $\chi(T)$

b Determined from Rietveld refinement against Polaris data collected at $50 \mathrm{~K}$.

\section{Neutron Diffraction}

\section{Ambient temperature}

Medium resolution neutron diffraction measurements were performed on the Polaris instrument at room temperature. The focused and absorption corrected data from all 5 of Polaris' detector banks were subsequently analysed using Rietveld refinement, the results of which are shown in Table II. During the refinements the occupancy of the mixed transition metal sites were constrained to unity but the ratios allowed to refine. We found no evidence for oxygen non-stoichiometry ( $\mathrm{O}$ site occupancies did not change significantly from unity during trial refinements and were subsequently fixed at this value). The material with $x=0.2$ was shown to be or- 

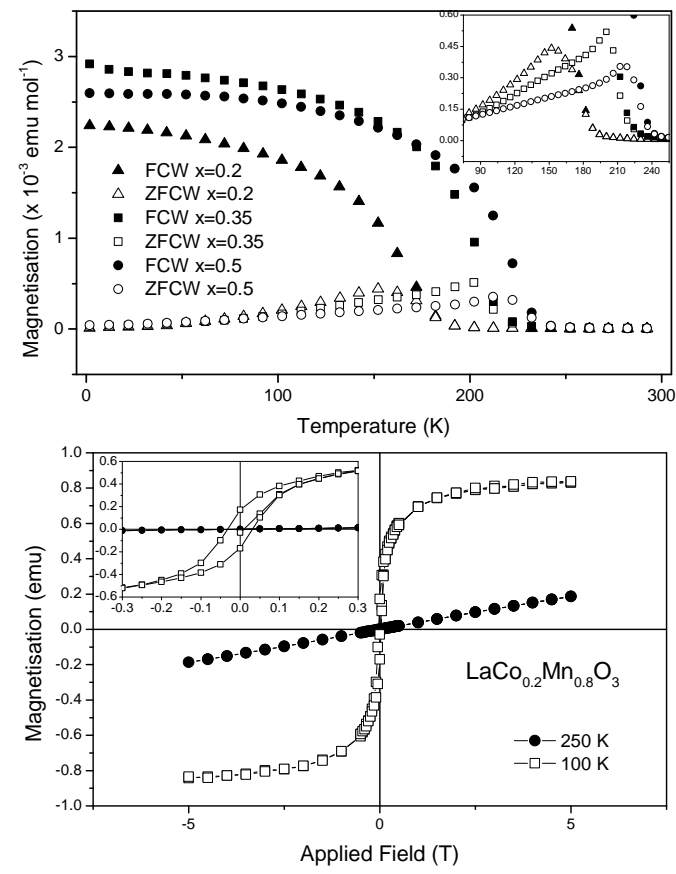

FIG. 2. Upper: Magnetisation of $\mathrm{LaCo}_{x} \mathrm{Mn}_{1-x} \mathrm{O}_{3}$ (for $x=$ $0.2,0.35$ and 0.5$)$ as a function of temperature, under both zero-field cooled warming (ZFCW) and field cooled warming (FCW) conditions. Insert shows in detail the ZFCW behaviour close to the determined Curie temperature. Lower: Typical magnetisation versus applied field loops at temperatures above and below $\mathrm{T}_{\mathrm{C}}$ for compositions $x=0.2,0.35$ and 0.5. No hysteresis is seen above $\mathrm{T}_{\mathrm{C}}$ but below, an open loop consistent with ferromagnetic ordering is seen. Insert shows the data close to zero applied field.

thorhombic with space group Pbnm, in agreement with previous reports and with X-ray diffraction. In this structure both transition metal (TM) ions are statistically distributed over a single crystallographic site, and therefore the obtained TM-O distances represent an average value which cannot provide information on the occurrence of (or degree of) charge ordering, or the assignment of possible oxidation states. Of particular interest is the refinement of the sample with the composition $\mathrm{LaCo}_{0.35} \mathrm{Mn}_{0.65} \mathrm{O}_{3}$. In this case, the Pbnm structure proved unsatisfactory and the data were in fact best fit with a monoclinic structure, space group $P 2_{1} / n$, which has previously only been reported for the sample with $x=0.5 .^{14}$ The improvement in goodness-of-fit parameters is detailed in Table II, and the fit itself is shown in Figure 4. This monoclinic structure provides two distinct sites for the transition metal ions and refining the site occupancies on the $2 c$ and $2 d$ Wyckoff sites reveals a high degree of order and hence different TM-O distances for each cation can be determined. For the samples with composition $x=0.75$ and 0.9 the symmetry was found to be rhombohedral with the space group $R \overline{3} c$ which again
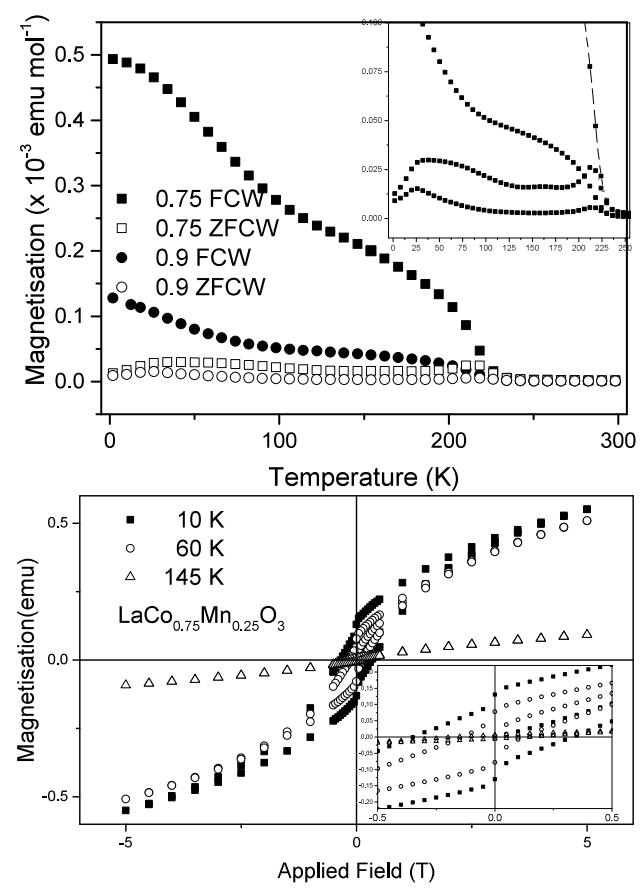

FIG. 3. Upper: Magnetisation of $\mathrm{LaCo}_{x} \mathrm{Mn}_{1-x} \mathrm{O}_{3}$ (for $x=$ $0.75,0.9)$ as a function of temperature, under both zero-field cooled warming (ZFCW) and field cooled warming (FCW) conditions. Insert shows in detail the unusual behaviour below $250 \mathrm{~K}$. Lower: Magnetisation versus applied field loops for $x=0.75$ at temperatures above and below the observed features ZFCW data. No hysteresis is seen above $145 \mathrm{~K}$, and below this temperature the curves resemble those expected for a ferromagnetic material, but do not approach saturation, even at $\mathrm{H}=5.0 \mathrm{~T}$.

does not allow any transition metal site ordering and only provides an average TM-O distance.

The reduced lattice parameters for the $\mathrm{LaCo}_{x} \mathrm{Mn}_{1-x} \mathrm{O}_{3}$ series, as determined from mediumresolution neutron diffraction data, are shown in Figure 5 , along with those reported by Autret. ${ }^{10}$ Interestingly we do not observe a pseudocubic lattice (i.e. $\left.a_{p c} \simeq b_{p c} \simeq c_{p c}\right)$ for $x=0.2$ in the Pbnm space group, and instead found that for $x \leq 0.5$ the reduced lattice parameters changed only slightly, with $a_{p c} \gg c_{p c}>b_{p c}$ in all cases. The rhombohedral lattice parameter reduces with $x$, in good agreement with previous reports. The average bond distances show a gradual change from that determined for $\mathrm{LaMnO}_{3}$ to $\mathrm{LaCoO}_{3}$ with increasing cobalt content as shown in Figure 6 and detailed in Table II. 
TABLE II: Structural parameters for $\mathrm{LaCo}_{x} \mathrm{Mn}_{1-x} \mathrm{O}_{3}$ as determined by Rietveld refinement against Polaris data. Parameters for both orthorhombic and monoclinic fits are shown for the sample with $x=0.35$.

\begin{tabular}{|c|c|c|c|c|c|c|c|}
\hline & & $x=0.2$ & $x=0.35$ & $x=0.35$ & $x=0.5$ & $x=0.75$ & $x=0.9$ \\
\hline & Space group & Pbnm & Pbnm & $P 2_{1} / n$ & $P 2_{1} / n$ & $R \overline{3} c$ & $R \overline{3} c$ \\
\hline & $\mathrm{a}(\AA)$ & $5.5297(1)$ & $5.5258(2)$ & $5.5259(1)$ & $5.52464(9)$ & $5.4071(2)$ & $5.3846(1)$ \\
\hline & b $(\AA)$ & $5.4884(1)$ & $5.4844(2)$ & $5.4840(1)$ & $5.48302(9)$ & $=\mathrm{a}$ & $=\mathrm{a}$ \\
\hline & c $(\AA)$ & $7.7774(2)$ & $7.7714(2)$ & $7.7709(1)$ & $7.7717(1)$ & $=\mathrm{a}$ & $=\mathrm{a}$ \\
\hline & $\alpha\left(^{\circ}\right)$ & 90 & 90 & 90 & 90 & $60.828(1)$ & $60.801(1)$ \\
\hline & $\beta\left({ }^{\circ}\right)$ & 90 & 90 & $89.930(3)$ & $89.898(3)$ & $=\alpha$ & $=\alpha$ \\
\hline & $\gamma\left({ }^{\circ}\right)$ & 90 & 90 & 90 & 90 & $=\alpha$ & $=\alpha$ \\
\hline & ${ }^{w} R_{p}(\%)$ & 2.09 & 3.03 & 2.00 & 2.09 & 2.87 & 3.67 \\
\hline & $\mathrm{R}_{p}(\%)$ & 2.44 & 3.01 & 2.16 & 2.67 & 3.45 & 5.23 \\
\hline \multirow[t]{6}{*}{$\mathrm{La}$} & Wyckoff site & $4 c$ & $4 c$ & $4 e$ & $4 e$ & $2 a$ & $2 a$ \\
\hline & $\mathrm{x}$ & $0.9947(2)$ & $0.9916(2)$ & $0.0041(1)$ & $0.0057(1)$ & 0.25 & 0.25 \\
\hline & $\mathrm{y}$ & $0.0204(1)$ & $0.0212(1)$ & $0.02096(8)$ & $0.02160(9)$ & 0.25 & 0.25 \\
\hline & $\mathrm{z}$ & 0.25 & 0.25 & $0.2485(2)$ & $0.2438(1)$ & 0.25 & 0.25 \\
\hline & $\mathrm{U}_{\text {iso }}\left(\AA^{2}\right)$ & $0.00775(8)$ & $0.0069(1)$ & $0.00608(7)$ & $0.00400(7)$ & $0.00578(8)$ & $0.00456(8)$ \\
\hline & S.O.F. & 1 & 1 & 1 & 1 & 1 & 1 \\
\hline \multirow[t]{9}{*}{$\mathrm{Co} / \mathrm{Mn}(1)$} & Wyckoff site & $4 b$ & $4 b$ & $2 c$ & $2 c$ & $2 b$ & $2 b$ \\
\hline & $\mathrm{x}$ & 0.5 & 0.5 & 0 & 0 & 0 & 0 \\
\hline & $\mathrm{y}$ & 0 & 0 & 0.5 & 0.5 & 0 & 0 \\
\hline & $\mathrm{z}$ & 0 & 0 & 0 & 0 & 0 & 0 \\
\hline & $\mathrm{U}_{i s o}\left(\AA^{2}\right)$ & $0.0024(1)$ & $0.0003(3)$ & $0.001(2)$ & 0.005 & 0.005 & 0.005 \\
\hline & S.O.F (Co) & $0.213(1)$ & $0.350(2)$ & $0.690(2)$ & $0.881(2)$ & $0.760(1)$ & $0.920(1)$ \\
\hline & $\mathrm{Co} / \mathrm{Mn}-\mathrm{O} 1(\AA)$ & $2 \times 1.97817(3)$ & $2 \times 1.9774(2)$ & $2 \times 2.0247(14)$ & $2 \times 2.0371(9)$ & $6 \times 1.94496(7)$ & $6 \times 1.93485(6)$ \\
\hline & $\mathrm{Co} / \mathrm{Mn}-\mathrm{O} 2(\AA)$ & $\begin{array}{l}2 \times 1.97817(3) \\
2 \times 1.97654(2)\end{array}$ & $\begin{array}{l}2 \times 1.9774(2) \\
2 \times 1.9781(7)\end{array}$ & $2 \times 2.0238(15)$ & $2 \times 2.0360(9)$ & - & - \\
\hline & Co/Mn - O3 $(\AA)$ & - & - & $2 \times 2.033(2)$ & $2 \times 2.0491(9)$ & - & - \\
\hline \multirow[t]{9}{*}{$\mathrm{Co} / \mathrm{Mn}(2)$} & Wyckoff site & - & - & $2 d$ & $2 d$ & - & - \\
\hline & $\mathrm{x}$ & - & - & 0.5 & 0.5 & - & - \\
\hline & $\mathrm{y}$ & - & - & 0.0 & 0.0 & - & - \\
\hline & $\mathrm{z}$ & - & - & 0.0 & 0.0 & - & - \\
\hline & $\mathrm{U}_{\text {iso }}\left(\AA^{2}\right)$ & - & - & $0.0032(3)$ & 0.005 & - & - \\
\hline & S.O.F (Co) & - & - & 0 & $0.119(2)$ & - & - \\
\hline & $\mathrm{Co} / \mathrm{Mn}-\mathrm{O} 1(\AA)$ & - & - & $2 \times 1.9259(14)$ & $2 \times 1.9207(9)$ & - & - \\
\hline & $\mathrm{Co} / \mathrm{Mn}-\mathrm{O} 2(\AA)$ & - & - & $2 \times 1.9225(15)$ & $2 \times 1.9116(9)$ & - & - \\
\hline & $\mathrm{Co} / \mathrm{Mn}-\mathrm{O} 3(\AA)$ & - & - & $2 \times 1.920(2)$ & $2 \times 1.9030(9)$ & - & - \\
\hline \multirow[t]{6}{*}{ O1 } & Wyckoff site & $4 c$ & $4 c$ & $4 e$ & $4 e$ & $6 e$ & $6 e$ \\
\hline & $\mathrm{x}$ & $0.0656(1)$ & $0.0664(2)$ & $0.2836(3)$ & $0.2879(2)$ & $-0.19760(4)$ & $-0.19962(4)$ \\
\hline & $\mathrm{y}$ & $0.4948(2)$ & $0.4950(2)$ & $0.2712(3)$ & $0.2732(2)$ & $0.69760(4)$ & $0.69962(4)$ \\
\hline & $\mathrm{z}$ & 0.25 & 0.25 & $0.0335(3)$ & $0.0347(2)$ & 0.25 & 0.25 \\
\hline & $\mathrm{U}_{\text {iso }}\left(\AA^{2}\right)$ & $0.0087(2)$ & $0.0077(2)$ & $0.0087(4)$ & $0.0059(2)$ & $0.00817(6)$ & $0.00755(7)$ \\
\hline & S.O.F. & 1 & 1 & 1 & 1 & 1 & 1 \\
\hline \multirow[t]{6}{*}{$\mathrm{O} 2$} & Wyckoff site & $8 d$ & $8 d$ & $4 e$ & $4 e$ & - & - \\
\hline & $\mathrm{x}$ & $0.7285(1)$ & $0.7283(1)$ & $0.2631(2)$ & $0.2611(1)$ & - & - \\
\hline & $\mathrm{y}$ & $0.2724(1)$ & $0.2712(1)$ & $0.2765(4)$ & $0.2775(2)$ & - & - \\
\hline & $\mathrm{z}$ & $0.03511(7)$ & $0.03751(8)$ & $0.4633(2)$ & $0.4621(2)$ & - & - \\
\hline & $\mathrm{U}_{\text {iso }}\left(\AA^{2}\right)$ & $0.0106(1)$ & $0.0088(1)$ & & & & \\
\hline & S.O.F. & 1 & 1 & 1 & 1 & - & - \\
\hline \multirow[t]{6}{*}{ O3 } & Wyckoff site & - & - & $4 e$ & $4 e$ & - & - \\
\hline & $\mathrm{x}$ & - & - & $0.5652(1)$ & $0.5650(1)$ & - & - \\
\hline & $\mathrm{y}$ & - & - & $-0.0062(1)$ & $-0.0053(1)$ & - & - \\
\hline & $\mathrm{z}$ & - & - & $0.2425(3)$ & $0.2404(1)$ & - & - \\
\hline & $\mathrm{U}_{\text {iso }}\left(\AA^{2}\right)$ & - & - & $0.0082(2)$ & $0.0073(1)$ & - & - \\
\hline & S.O.F. & - & - & 1 & 1 & - & - \\
\hline
\end{tabular}

For the $x=0.75$ and 0.9 composition samples, high resolution neutron powder diffraction data was measured on the HRPD instrument. Data from the $90^{\circ}$ bank of detectors were exactly comparable to those measured on Polaris at room temperature. For the $x=0.9$ composition, data from the $168^{\circ}$ backscattering detector bank revealed shoulders on each of the reflections. The best fit to these data was obtained with a mixture of $\mathrm{LaCo}_{0.85} \mathrm{Mn}_{0.15} \mathrm{O}_{3}$ and $\mathrm{LaCoO}_{2.95}$. The structural parameters of each phase were refined and are shown in Table III. Taking into account the phase fractions of both phases the total ratio of Co:Mn in the sample remains 0.9:0.1, as intended. Since this phase separation is only observable by high resolution diffraction, we suggest that the limit of the $\mathrm{LaCo}_{x} \mathrm{Mn}_{1-x} \mathrm{O}_{3}$ solid solution is reached at $x=0.85$.

\section{Low temperature}

Low temperature neutron diffraction data were also obtained for each of the samples on the medium resolution Polaris diffractometer. Datasets were obtained at $50 \mathrm{~K}$ (significantly below the magnetic ordering temperature) with a view to obtaining details of the magnetic structure.

For the compositions $x=0.2,0.35$ and 0.5 enhanced intensity of the $110 / 002$ and $112 / 020 / 200$ reflections is consistent with ferromagnetic ordering along the $c$-axis, magnetic space group $P b^{\prime} n ' m$, similar to our previous report. ${ }^{25}$ The refined ordered moments are reported in Table I. We stress that due to limitations of data quality and instrument resolution we used the orthorhombic symmetry to model the magnetic structure of all three samples, despite the nuclear structures of the $x=0.35$ 


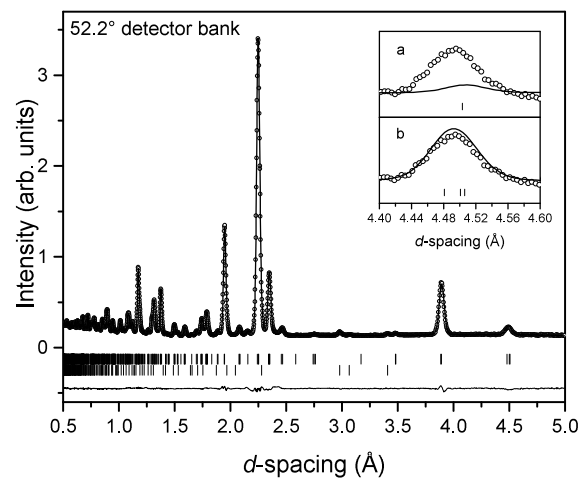

FIG. 4. Result of Rietveld refinement of the $x=0.35$ sample using the monoclinic $P 2_{1} / n$ structure. Inset a) shows the poor fit of the orthorhombic model to the data in the region of the 101 reflection, and b) the significant improvement on allowing the 101 and $10 \overline{1}$ to split via the monoclinic beta angle being non-90 degrees, but also the presence of the 011 reflection which is a systematic absence in space group Pbnm.

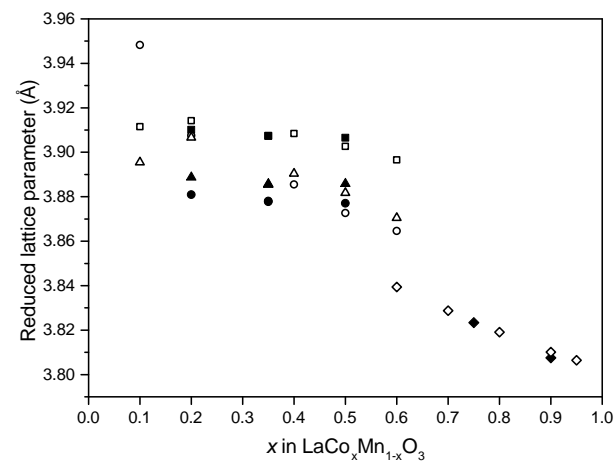

FIG. 5. Determined lattice parameters as a function of composition for the series $\mathrm{LaCo}_{x} \mathrm{Mn}_{1-x} \mathrm{O}_{3}$ for the orthorhombic and rhombohedral unit cells. Shown are the three equivalent lattice parameters $\left(a_{p c}\right)$ for the cubic $\mathrm{ABX}_{3}$ perovskite (where, for the orthorhombic lattice parameters, $a=\sqrt{2} a_{p c}$, $b=2 b_{p c}$ and $c=2 c_{p c}$ and for the rhombohedral unit cell $a$ $=\sqrt{2} a_{p c}$ ). In this figure, values for $a_{p c}, b_{p c}$ and $c_{p c}$ for the orthorhombic cells are shown as solid squares, triangles and circles, respectively, and $a_{p c}$ for the rhombohedral cells are shown as solid diamonds. The smaller, open symbols are the reported values from Autret et al. ${ }^{10}$

and 0.5 samples being better described by the monoclinic structure. The magnetic structure of $\mathrm{LaCo}_{0.5} \mathrm{Mn}_{0.5} \mathrm{O}_{3}$ was recently explored by Orayech et al, ${ }^{22}$ who identified three potential, though indistinguishable, ferromagnetic models in space groups $P 2_{1} / n$ and $P 2_{1}^{\prime} / n^{\prime}$.

In contrast, for the $x=0.75$ and 0.9 samples no extra reflections are observed, even on cooling to $4 \mathrm{~K}$. The lack of reflections with magnetic origin suggests that there is no long range magnetic ordering in the materials at this temperature. This is consistent with the observation of

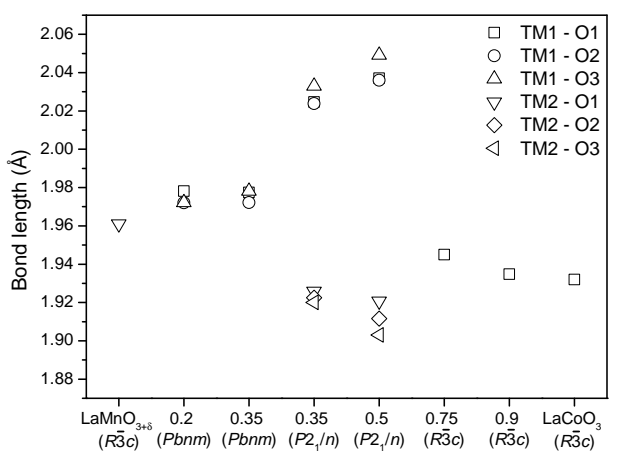

FIG. 6. Bond lengths obtained from Rietveld refinements for each member of the $\mathrm{LaCo}_{x} \mathrm{Mn}_{1-x} \mathrm{O}_{3}$ series. For the rhombohedral $(R \overline{3} c)$ and orthorhombic $(P b n m)$ structures only an average $\mathrm{Co} / \mathrm{Mn}-\mathrm{O}$ distance is determined as a result of site disorder. However, for the monoclinic $P 2_{1} / n$ structure distinct $\mathrm{Co}-\mathrm{O}(\sim 2.02 \AA)$ and $\mathrm{Mn}-\mathrm{O}(\sim 1.92 \AA)$ bond distances are determined as the transition metal ions Co and Mn are shown to be ordered and preferentially sited on the $2 \mathrm{c}$ and $2 \mathrm{~d}$ Wyckoff sites, respectively (see Table II).

TABLE III. Structural parameters for the two phases found for the $x=0.9$ sample. Overall goodness-of-fit parameters are: $\mathrm{wR}_{p}=4.33 \%, \mathrm{R}_{p}=2.45 \%$.

\begin{tabular}{llll}
\hline \hline & & Phase 1 & Phase 2 \\
& Weight fraction & $0.504(13)$ & $0.496(13)$ \\
Space group & $R \overline{3} c$ & $R \overline{3} c$ \\
$a=b=c(\AA)$ & $5.3950(4)$ & $5.38401(9)$ \\
La & $\alpha=\beta=\gamma\left({ }^{\circ}\right)$ & $60.801(2)$ & $60.792(1)$ \\
& Wyckoff site & $2 a$ & $2 a$ \\
& $x$ & 0.25 & 0.25 \\
& $y$ & 0.25 & 0.25 \\
& $z$ & 0.25 & 0.25 \\
& $\mathrm{U}_{i s o}\left(\AA^{2}\right)$ & 0.003 & $0.0017(7)$ \\
Co/Mn & 1 & 1 \\
& Wyckoff site & $2 b$ & $2 b$ \\
& $x$ & 0 & 0 \\
& $y$ & 0 & 0 \\
& $z$ & 0 & 0 \\
& $\mathrm{U}_{i s o}\left(\AA^{2}\right)$ & $0.002(5)$ & $0.005(2)$ \\
S.O.F. $($ Co $)$ & $0.85(1)$ & 1 \\
& Wyckoff site & $6 e$ & $6 e$ \\
& $x$ & $-0.1988(3)$ & $-0.2010(3)$ \\
& $y$ & $0.6988(3)$ & $0.7010(3)$ \\
& $z$ & 0.25 & 0.25 \\
& $\mathrm{U}_{\text {iso }}\left(\AA^{2}\right)$ & $0.0015(7)$ & $0.0053(7)$ \\
& S.O.F. & 1 & $0.98(1)$ \\
\hline \hline
\end{tabular}

only a weak ferromagnetic signal in the DC susceptibility. However, we do note that if $k=0$, the additional intensity will be on the nuclear peaks and hence if small, masked by atomic displacement parameters. Despite being statistically acceptable, careful visual inspection of these fits revealed some discrepancies in peak shape and position that could not be resolved using the mediumresolution data. Hence, a high-resolution study was carried out on the HRPD instrument. We note briefly that for $x=0.9$, the two-phase mixture described above for the room temperature data also produced a satisfactory fit to the $4 \mathrm{~K}$ data. In contrast, the high resolution 
neutron diffraction study of the sample with composition $x=0.75$ at low temperature shows some interesting features. There are significant shoulders to the reflections which are particularly observable around $2.25 \AA$. We have made significant effort to fit this dataset with the rhombohedral structure in combination with other possible oxides containing $\mathrm{La}, \mathrm{Co}$ and $\mathrm{Mn}$ including the parent perovskites of the series. None of these materials were able to satisfactorily fit the diffraction pattern. To this end we tried a lower symmetry crystalline structure for the majority perovskite phase. This was achieved initially by reducing the symmetry to triclinic $P \overline{1}$, with $a=5.39302(28) \AA, b=5.37840(58) \AA, c=5.39303(28)$ $\AA, \alpha=61.003(3)^{\circ}, \beta=61.003(5)^{\circ}$ and $\gamma=61.003(3)^{\circ}$. We note this metric has $a=c \neq b, \alpha \sim \beta \sim \gamma$. Upon conversion to a more standard monoclinic perovskite space group, $I 2 / a$, the lattice parameters refine as $a=7.670(7)$ $\AA, b=5.4729(5) \AA, c=5.3821(5) \AA$ and $\beta=91.082(4)^{\circ}$. The quality of fit by Rietveld refinement was given as $\mathrm{wR}_{p}=3.62 \%$ (which should be compared with the value of $4.64 \%$ that was obtained using the rhombohedral setting). Whilst not fully satisfactory the fit did improve, as evidenced by the splitting of the 210 reflection from $R \overline{3} c$ at $\sim 2.31 \AA$ to $11 \overline{2}, 310$ and 121 at $\sim 2.31 \AA$ and $12 \overline{1}$ at $\sim 2.33 \AA$ in $I 2 / a$ (Figure 7 ). In this model the Co and $\mathrm{Mn}$ are disordered on the $4 b$ Wyckoff site, with TM-O bond lengths ranging from $1.855 \AA$ to $2.016 \AA$, entirely consistent with $\mathrm{Mn}-\mathrm{O}$ and $\mathrm{Co}-\mathrm{O}$ bond lengths of varying TM oxidation states. We suggest that this complicated monoclinic structure could be used as a starting point for future studies of this material and may start to account for the unusual magnetic behaviour of the materials in this series with high cobalt content.

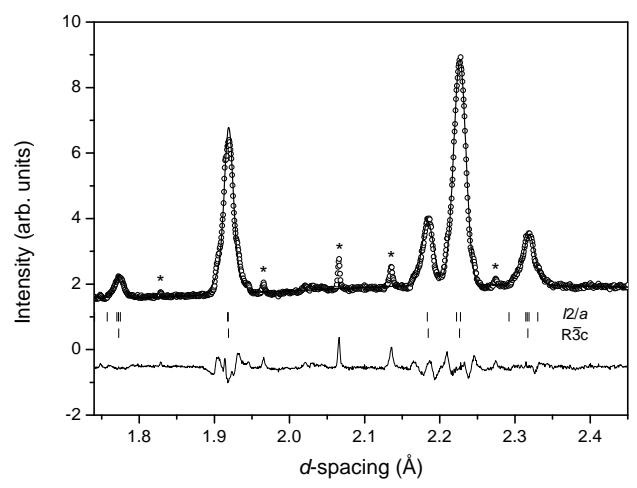

FIG. 7. Selected region of the Rietveld refinement of the monoclinic $(I 2 / a)$ model for $\mathrm{LaCo}_{0.75} \mathrm{Mn}_{0.25} \mathrm{O}_{3}$ using high resolution neutron data obtained from HRPD. Also shown are the allowed reflections for the rhombohedral $(R \overline{3} c)$ structure, which highlights the improvement in fit obtained by reducing the symmetry of the model. The reflections marked with asterisks are attributed to the cryostat background.

The complete lack of magnetic Bragg reflections combined with the weakly ferromagnetic nature of the $x=$ 0.75 sample prompted a detailed investigation of this ma- terial using $\mathrm{AC}$ susceptibility. The real part of the $\mathrm{AC}$ susceptibility, $\chi^{\prime}$, as shown in Figure 8(a), has a peak at $\sim 218 \mathrm{~K}$, and a broad maxima at $\sim 25 \mathrm{~K}$. The imaginary part of the AC susceptibility, $\chi^{\prime \prime}$, is non-zero at temperatures lower than about $235 \mathrm{~K}$, again contains a peak at $218 \mathrm{~K}$, and the broad maxima at low temperature has sharpened into a second peak. The $218 \mathrm{~K}$ feature does not exhibit a frequency dependence (see the upper panel of Figure 9) which implies that it arises from a ferromagnetic ordering transition. Given the lack of magnetic Bragg peaks it is clear that the coherence length of this ordering is rather small. Conversely, the position of peak at $\sim 25 \mathrm{~K}$ does exhibit a clear frequency dependence across the range measured $(250-10000 \mathrm{~Hz})$. Such a dependence, observed at the relatively low frequencies used here, is indicative of some form of frustrated and/or glassy ordering. ${ }^{32}$ The magnitude of the frequency dependence can be approximated by the relationship $\Delta \mathrm{T}_{\mathrm{f}} / \mathrm{T}_{\mathrm{f}} \Delta(\log \omega)$, as per Mydosh. ${ }^{32}$ Values for $\mathrm{T}_{\mathrm{f}}$ at each frequency were obtained by fitting a quadratic function of the form $\chi^{\prime \prime}=A+B\left(\mathrm{~T}-\mathrm{T}_{\mathrm{f}}\right)^{2}$ to the peaks in the temperature dependence of $\chi^{\prime \prime}$, and a value of 0.094 was obtained for the Mydosh parameter. This value is larger than those observed for canonical spin glasses (which tend to lie in the range $5 \times 10^{-3}-2 \times 10^{-2}$ ) and significantly smaller than those expected for superparamagnets $(\gg 0.1) .{ }^{32}$ Some specific examples include 0.013 for double perovskite $\mathrm{SrLaCoReO}_{6},{ }^{33} 0.022$ for $\mathrm{Sr}_{2} \mathrm{FeTeO}_{6},{ }^{34}$ and 0.085 for $\mathrm{LaCo}_{0.5} \mathrm{Mn}_{0.5} \mathrm{O}_{3} .{ }^{35}$ Intermediate values of the Mydosh parameter are commonly observed in socalled cluster glasses where weakly-interacting ferromagnetic clusters are randomly oriented. ${ }^{36-38}$ The magnitude of the frequency dependence can, in general, be related to the strength of interaction between the magnetic entities. ${ }^{37,39}$ In the case of an ordered ferromagnetic system, this interaction (between atoms) is strong and hence very high frequencies (well outside the range explored here) are required to see any frequency dependence. Conversely, an idealised superparamagnetic system consisting of non-interacting particles (for example, $\alpha-\left(\mathrm{Ho}_{2} \mathrm{O}_{3}\left(\mathrm{~B}_{2} \mathrm{O}_{3}\right)\right)^{32}$ has a much higher sensitivity to frequency and thus a larger value of the Mydosh parameter. To further characterise the dynamics of the magnetic behaviour of this material, the low-temperature peak in the AC susceptibility was probed at several additional frequencies, and an attempt was made to fit the frequency dependence of $T_{f}$ to the Vogel-Fulcher law, but the fitted parameters were unphysical. Therefore the data were fitted using the Ogielski scaling relation, ${ }^{45,46}$ $\mathrm{T}_{\mathrm{f}}=\mathrm{T}_{\mathrm{g}}\left[1+\left(f \tau_{0}\right)^{1 / z \nu}\right]$, where $\mathrm{T}_{g}$ is the "phase transition" temperature, equivalent to extrapolating $\mathrm{T}_{\mathrm{f}}$ to 0 $\mathrm{Hz}, \tau_{0}$ is a characteristic time of the system and $z \nu$ a critical exponent. The fit is shown in figure 9 and the values obtained were $\mathrm{T}_{\mathrm{g}}=23.9(1) \mathrm{K}, \tau_{0}=4.8(7) \times 10^{-7}$ $\mathrm{s}$ and $z \nu=2.0(1)$. The characteristic time is indicative of rather slow spin flipping dynamics, and indeed is rather higher than those typically obtained for canonical spin glasses, but well within the range reported for re-entrant 
spin glasses and cluster glasses, such as $\mathrm{Nd}_{5} \mathrm{Ge}_{3}\left(10^{-7}\right.$ s), ${ }^{47} \mathrm{La}_{0.95} \mathrm{Sr}_{0.05} \mathrm{CoO}_{3}\left(10^{-10} \mathrm{~s}\right){ }^{48} \mathrm{LaCo}_{0.5} \mathrm{Ni}_{0.5} \mathrm{O}_{3}\left(10^{-5}\right.$ s) ${ }^{40}$ and $\mathrm{LaMn}_{0.5} \mathrm{Fe}_{0.5} \mathrm{O}_{3}\left(10^{-5} \mathrm{~s}\right) .{ }^{49}$ The evidence from AC susceptibility overwhelmingly suggests that the behaviour of $\mathrm{LaCo}_{0.75} \mathrm{Mn}_{0.25} \mathrm{O}_{3}$ can be classified as a cluster glass. This behaviour is very similar to the re-entrant spin-glass behaviour observed in $\mathrm{LaCo}_{0.5} \mathrm{Ni}_{0.5} \mathrm{O}_{3},{ }^{40}$ and the "glassy ferromagnetism" reported several times for $\mathrm{La}_{1-x} \mathrm{Sr}_{x} \mathrm{CoO}_{3} .{ }^{41,42}$
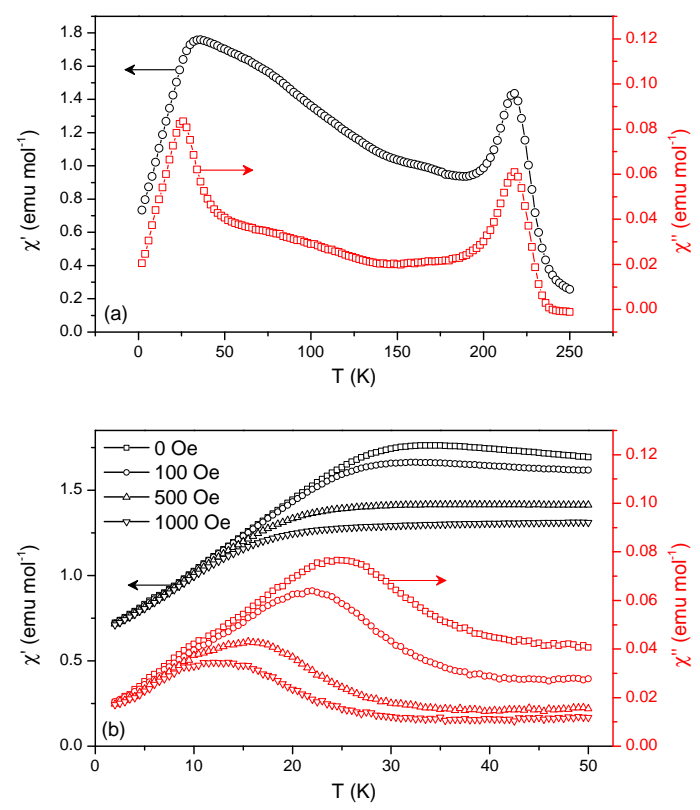

FIG. 8. (a) Real and imaginary parts of the AC susceptibility of $\mathrm{LaCo}_{0.75} \mathrm{Mn}_{0.25} \mathrm{O}_{3}$ as a function of temperature $(2-250$ $\mathrm{K}$ ), measured at $1.016 \mathrm{kHz}$ with $0 \mathrm{DC}$ bias field. (b) The 0 $50 \mathrm{~K}$ region of the $\mathrm{AC}$ susceptibility measured at $255 \mathrm{~Hz}$ in various DC bias fields.

\section{E. Phase Diagram}

In Figure 10 we present the structural and magnetic phase diagram of the solid solution $\mathrm{LaCo}_{x} \mathrm{Mn}_{1-x} \mathrm{O}_{3}$. For each of the materials the synthetic conditions were identical. At room temperature, a composition-dependent transition from orthorhombic to monoclinic and then rhombohedral is observed. The monoclinic symmetry, as reported previously for $x=0.5,{ }^{14}$ and for the first time herein for $x=0.35$, is a result of transition metal cation ordering. Also shown is the orthorhombic/monoclinic to rhombohedral boundary and regions of phase coexistence. The regions of phase co-existence are quite large as a result of the strain inherent in these samples (as indicated by the peak width in the diffraction patterns, especially noticeable if you compare that to the parent materials synthesised by the same routes). The extrapolated second order phase transition to the metrically cubic phase is also shown (this transition being well below the melting points for the parent compounds (2013 K
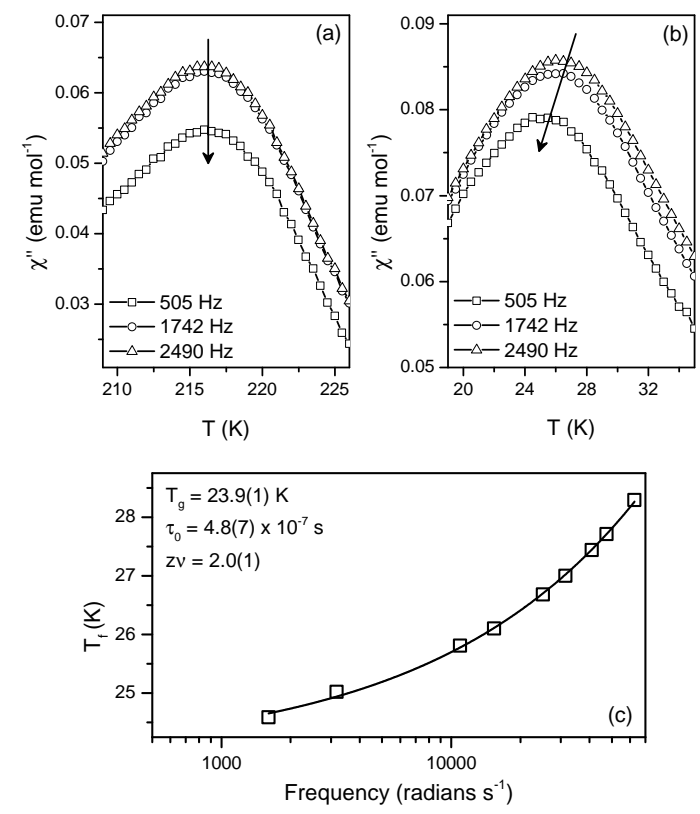

FIG. 9. (a) and (b) Close-up views of the two peaks in the imaginary part of the AC susceptibility of $\mathrm{LaCo}_{0.75} \mathrm{Mn}_{0.25} \mathrm{O}_{3}$ at three different frequencies. The frequency dependence in the position of the $\sim 25 \mathrm{~K}$ peak is clear. Arrows are a guide to the eye. (c) Result of fitting the frequency dependence of $\mathrm{T}_{\mathrm{f}}$ with the conventional critical "slowing down" model.

and $2153 \mathrm{~K}$ for $\mathrm{LaCoO}_{3}$ and $\mathrm{LaMnO}_{3}$ respectively. ${ }^{43,44}$. Also shown are the ferromagnetic ordering temperatures for each material studied as described in Section IIID and in tabulated in Table I.

\section{CONCLUSIONS}

We have investigated the structural and magnetic phase diagram of the solid solution $\mathrm{LaCo}_{x} \mathrm{Mn}_{1-x} \mathrm{O}_{3}$ using $\mathrm{X}$-ray and neutron diffraction and magnetometry techniques. We have shown that for the composition $x=0.35$ the transition metals are site ordered and the sample crystallises with a monoclinic crystallographic structure $P 2_{1} / n$. We have also confirmed the disordered nature of the transition metal ions in the compositions $x=0.2$, 0.75 and 0.9 . Phase separation is observed in high resolution neutron diffraction for $x=0.9$ and suggests that the solid solution limit is in fact $x=0.85$. Structurally we have also presented evidence to suggest that the rhombohedral $x=0.75$ composition at low temperature transforms into a monoclinic structure.

We have also been able to map out a potential phase diagram as a function of temperature and have shown that there are a series of temperature-driven transitions from orthorhombic/monoclinic to rhombohedral and then to cubic. Transition temperatures are composition dependent. Further evidence is provided (by neutron diffraction and $\mathrm{AC}$ and $\mathrm{DC}$ magnetometry methods) that the 


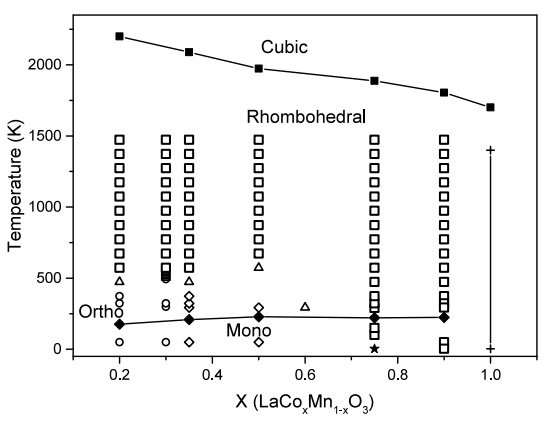

FIG. 10. Phase diagram of the series $\mathrm{LaCo}_{x} \mathrm{Mn}_{1-x} \mathrm{O}_{3}$ series as a function of temperature. The points where the orthorhombic symmetry materials exist are shown as open circles (o). Regions where rhombohedral symmetry exist are shown by open squares $(\square)$. Regions of phase co-existence (both orthorhombic/monoclinic and rhombohedral symmetry) shown as open triangles $(\triangle)$. Monoclinic regions are shown by open diamond $(\diamond)$. The extrapolated transition temperature from rhombohedral to pseudo cubic symmetry is shown by the joined solid squares ( $\mathbf{\square})$. The rhombohedral phase of $\mathrm{LaCoO}_{3}$ has been shown to exist from $4-1400 \mathrm{~K}$ by Thornton et al and is shown by the crosses $(+)$, data is also shown for the $x=0.33$ sample from previous work. ${ }^{7,25}$ The Curie temperature for each ferromagnetic transition is also shown as a solid diamond ( ). Finally, the proposed monoclinic phase is shown as a solid star for the sample with the composition $\mathrm{x}=0.75$. nature of the low-temperature magnetic structure in the rhombohedral phases $(x=0.75$ and 0.9$)$ is glassy in nature. The ferromagnetic nature of the orthorhombic and monoclinic materials has also been confirmed by use of neutron diffraction.

\section{ACKNOWLEDGMENTS}

The authors acknowledge access to the ISIS neutron facility at STFC and Materials Characterisation Laboratory capabilities therein. We also acknowledge the cryogenic technical support given by Dave Bunce of ISIS. Finally, we are grateful to Alexandra Gibbs for useful discussions.

1 A. P. Ramirez, Journal of Physics: Condensed Matter 9, 8171 (1997).

${ }^{2}$ H. Zhu, P. Zhang, and S. Dai, ACS Catalysis 5, 6370 (2015).

3 J. Hu, L. Wang, L. Shi, and H. Huang, Electrochimica Acta 161, 115 (2015).

${ }^{4}$ X. Ge, B. Li, D. Wuu, A. Sumboja, T. An, T. S. A. Hor, Y. Zong, and Z. Liu, Journal of Molecular and Engineering Materials 03, 1540006 (2015).

${ }^{5}$ H. Liang, Y. Hong, C. Zhu, S. Li, Y. Chen, Z. Liu, and D. Ye, Catalysis Today 201, 98 (2013).

${ }^{6}$ K. Knizek, Z. Jirak, J. Hejtmanek, P. Henry, and G. Andre, J. Appl. Phys. 103, $07 B 703$ (2008), arXiv:0710.2759.

7 G. Thornton, B. C. Tofield, and A. W. Hewat, Journal of Solid State Chemistry 61, 301 (1986).

8 A. M. Glazer, Acta Crystallographica Section B 28, 3384 (1972).

9 J. Rodriguez-Carvajal, M. Hennion, F. Moussa, A. H. Moudden, L. Pinsard, and A. Revcolevschi, Physical Review B 57, 3189 (1998).

10 C. Autret, J. Hejtmánek, K. Knížek, M. Maryško, Z. Jirák, M. Dlouhá, and S. Vratislav, Journal of Physics: Condensed Matter 17, 1601 (2005).

11 P. Ganguly, N. Y. Vasanthacharya, C. N. R. Rao, and P. P. Edwards, Journal of Solid State Chemistry 54, 400 (1984).

$12 \mathrm{Y} . \mathrm{Wu}, \mathrm{Z}$. Yu, and S. Liu, Journal of Solid State Chemistry 112, 157 (1994).

13 J. B. Goodenough, A. Wold, R. J. Arnott, and N. Menyuk,
Physical Review 124, 373 (1961).

14 C. L. Bull, D. Gleeson, and K. S. Knight, Journal of Physics: Condensed Matter 15, 4927 (2003).

15 G. H. Jonker, Journal of Applied Physics 37 (1966).

16 I. O. Troyanchuk, L. S. Lobanovsky, D. D. Khalyavin, S. N. Pastushonok, and H. Szymczak, Journal of Magnetism and Magnetic Materials 210, 63 (2000).

17 M. Sikora, C. Kapusta, K. Knížek, Z. Jirák, C. Autret, M. Borowiec, C. Oates, V. Procházka, D. Rybicki, and D. Zajac, Physical Review B 73, 094426 (2006).

18 M. Maryško, Z. Jirák, K. Knížek, and C. Autret-Lambert, Journal of Magnetism and Magnetic Materials 322, 1392 (2010).

19 R. Dass and J. B. Goodenough, Physical Review B 67, 014401 (2003).

20 T. Burnus, Z. Hu, H. H. Hsieh, V. L. J. Joly, P. A. Joy, M. W. Haverkort, H. Wu, A. Tanaka, H.-J. Lin, C. T. Chen, and L. H. Tjeng, Physical Review B 77, 125124 (2008).

21 P. A. Joy, Y. B. Khollam, and S. K. Date, Phys. Rev. B 62, 8608 (2000).

22 B. Orayech, I. Urcelay-Olabarria, G. A. López, O. Fabelo, a. Faik, and J. M. Igartua, Dalton Trans. 44, 13867 (2015).

23 X. L. Wang, M. James, J. Horvat, and S. X. Dou, Superconductor Science and Technology 15, 427 (2002).

24 J. Krishna Murthy and A. Venimadhav, Journal of Applied Physics 111, 024102 (2012).

${ }^{25}$ C. L. Bull, H. Y. Playford, K. S. Knight, W. G. Marshall, G. B. G. Stenning, R. I. Smith, and Z. Hart, Journal of 
physics. Condensed matter : an Institute of Physics journal 27, 165401 (2015).

${ }^{26}$ C. J. Howard and H. T. Stokes, Acta Crystallographica Section B Structural Science 54, 782 (1998).

27 S. A. Hayward, F. D. Morrison, S. A. T. Redfern, E. K. H. Salje, J. F. Scott, K. S. Knight, S. Tarantino, A. M. Glazer, V. Shuvaeva, P. Daniel, M. Zhang, and M. A. Carpenter, Physical Review B 72, 054110 (2005).

28 M. A. Carpenter, C. J. Howard, B. J. Kennedy, and K. S. Knight, Physical Review B - Condensed Matter and Materials Physics 72, 1 (2005).

29 Y. Kobayashi, T. Mitsunaga, G. Fujinawa, T. Arii, M. Suetake, K. Asai, and J. Harada, J. Phys. Soc. Jpn. 69, 3468 (2000).

30 M. Korotin, S. Ezhov, I. Solovyev, V. Anisimov, D. Khomskii, and G. a. Sawatzky, Physical Review B 54, 5309 (1996), arXiv:9709060 [cond-mat].

31 M. W. Haverkort, Z. Hu, J. C. Cezar, T. Burnus, H. Hartmann, M. Reuther, C. Zobel, T. Lorenz, A. Tanaka, N. B. Brookes, H. H. Hsieh, H.-J. Lin, C. T. Chen, and L. H. Tjeng, Physical Review Letters 97, 176405 (2006).

32 J. A. Mydosh, Spin Glasses An Experimental Introduction (Taylor \& Francis, London, 1993).

33 C. M. Thompson, L. Chi, J. R. Hayes, A. M. Hallas, M. N. Wilson, T. J. S. Munsie, I. P. Swainson, A. P. Grosvenor, G. M. Luke, and J. E. Greedan, Dalton Trans. 44, 10806 (2015).

34 L. Ortega-San Martin, J. P. Chapman, L. Lezama, J. J. Saiz Garitaonandia, J. Sánchez Marcos, J. RodríguezFernández, M. I. Arriortua, and T. Rojo, Journal of Materials Chemistry 16, 66 (2006).

35 J. Krishna Murthy and A. Venimadhav, Journal of Applied Physics 113, 2011 (2013).
${ }^{36}$ V. K. Anand, D. T. Adroja, and A. D. Hillier, Physical Review B 85, 014418 (2012).

37 T. Chakrabarty, A. V. Mahajan, and S. Kundu, Journal of physics. Condensed matter : an Institute of Physics journal 26, 405601 (2014), arXiv:1408.5702.

38 A. Malinowski, V. L. Bezusyy, R. Minikayev, P. Dziawa, Y. Syryanyy, and M. Sawicki, Physical Review B 84, 024409 (2011).

39 K. Binder and A. P. Young, Reviews of Modern Physics 58, 801 (1986).

40 M. Viswanathan and P. S. A. Kumar, Physical Review B 80, 012410 (2009).

41 J. Wu and C. Leighton, Physical Review B 67, 174408 (2003).

42 R. Caciuffo, D. Rinaldi, G. Barucca, J. Mira, J. Rivas, M. A. Senaris-Rodriguez, P. G. Radaelli, D. Fiorani, and J. B. Goodenough, Physical Review B 59, 1068 (1999).

43 B. Gilbu, H. Fjellvå g, and A. Kjekshus, Acta Chemica Scandinavica 48, 37 (1994).

44 N. Q. Minh and T. Takahashi, Science and Technology of Ceramic Fuel Cells, edited by N. Q. M. Takahashi (Elsevier Science Ltd, Oxford, 1995).

45 A. T. Ogielski, Phys. Rev. B 32, 7384 (1985).

46 P. J. Baker, T. Lancaster, S. J. Blundell, M. L. Brooks, W. Hayes, D. Prabhakaran, and F. L. Pratt, Phys. B Condens. Matter 374-375, 47 (2006), arXiv:0506141 [condmat].

47 B. Maji, K. G. Suresh, and a. K. Nigam, J. Phys.: Condens. Matter 23, 506002 (2011).

48 D. N. H. Nam, R. Mathieu, P. Nordblad, N. V. Khiem, and N. X. Phuc, Phys. Rev. B 62, 8989 (2000).

49 K. De, M. Thakur, a. Manna, and S. Giri, J. Appl. Phys. 99, 013908 (2006). 